\title{
50 Percent Embryo Infective Dose per Milliliter
}

National Cancer Institute

\section{Source}

National Cancer Institute. 50 Percent Embryo Infective Dose per Milliliter. NCI Thesaurus.

Code 120847.

A potency unit for measuring infectious activity of a biologic product or an infectious agent preparation equal to the potency at which one milliliter of infectious material contains one 50 percent embryo infective dose. 